\title{
Differential Geometry of Rectifying Submanifolds
}

\author{
Bang-Yen Chen
}

(Communicated by Kazım İlarslan)

\begin{abstract}
A space curve in a Euclidean 3-space $\mathbb{E}^{3}$ is called a rectifying curve if its position vector field always lies in its rectifying plane. This notion of rectifying curves was introduced by the author in [Amer. Math. Monthly 110 (2003), no. 2, 147-152]. In this present article, we introduce and study the notion of rectifying submanifolds in Euclidean spaces. In particular, we prove that a Euclidean submanifold is rectifying if and only if the tangential component of its position vector field is a concurrent vector field. Moreover, rectifying submanifolds with arbitrary codimension are completely determined.
\end{abstract}

Keywords: Rectifying curve; rectifying submanifold.

AMS Subject Classification (2010): Primary: 53A07 ; Secondary: 53C40; 53C42.

\section{Introduction}

Let $\mathbb{E}^{3}$ denote Euclidean 3-space with its inner product $\langle$,$\rangle . Consider a unit-speed space curve x: I \rightarrow \mathbb{E}^{3}$, where $I=(\alpha, \beta)$ is a real interval. Let $\mathbf{x}$ denote the position vector field of $x$ and $\mathbf{x}^{\prime}$ be denoted by $\mathbf{t}$.

It is possible, in general, that $\mathbf{t}^{\prime}(s)=0$ for some $s$; however, we assume that this never happens. Then we can introduce a unique vector field $\mathbf{n}$ and positive function $\kappa$ so that $\mathbf{t}^{\prime}=\kappa \mathbf{n}$. We call $\mathbf{t}^{\prime}$ the curvature vector field, $\mathbf{n}$ the principal normal vector field, and $\kappa$ the curvature of the curve. Since $\mathbf{t}$ is of constant length, $\mathbf{n}$ is orthogonal to $\mathbf{t}$. The binormal vector field is defined by $\mathbf{b}=\mathbf{t} \times \mathbf{n}$, which is a unit vector field orthogonal to both $\mathbf{t}$ and $\mathbf{n}$. One defines the torsion $\tau$ by the equation $\mathbf{b}^{\prime}=-\tau \mathbf{n}$.

The famous Frenet-Serret equations are given by

$$
\left\{\begin{array}{l}
\mathbf{t}^{\prime}=\quad \kappa \mathbf{n} \\
\mathbf{n}^{\prime}=-\kappa \mathbf{t} \quad+\tau \mathbf{b} \\
\mathbf{b}^{\prime}=-\tau \mathbf{n} .
\end{array}\right.
$$

At each point of the curve, the planes spanned by $\{\mathbf{t}, \mathbf{n}\},\{\mathbf{t}, \mathbf{b}\}$, and $\{\mathbf{n}, \mathbf{b}\}$ are known as the osculating plane, the rectifying plane, and the normal plane, respectively.

From elementary differential geometry it is well known that a curve in $\mathbb{E}^{3}$ lies in a plane if its position vector lies in its osculating plane at each point, and lies on a sphere if its position vector lies in its normal plane at each point. In view of these basic facts, the author asked the following simple geometric question in [6]:

Question. When does the position vector of a space curve $\mathbf{x}: I \rightarrow \mathbb{E}^{3}$ always lie in its rectifying plane?

The author called such a curve a rectifying curve in [6]. The author derived many fundamental properties of rectifying curves. In particular, he completely classifies all rectifying curves in [6]. It is known that rectifying curves related with the notions constant-ratio curves and convolution (cf. $[3,4,5,7,8])$. Furthermore, the author and F. Dillen established in [11] a simple link between rectifying curves and the notion of centrodes in mechanics. Moreover, they showed in [11] that rectifying curves are indeed the extremal curves which satisfy the equality case of a general inequality. Since then rectifying curves have been studied by many authors, see $[1,14,15,16,17,18,19,20,21]$ among many others. For the most recent survey on rectifying curves, see [10]. 
In this article, we extend the notion of rectifying curves to the notion of rectifying submanifolds in a very natural way. Many fundamental properties of rectifying submanifolds are obtained. In particular, we prove that a Euclidean submanifold is rectifying if and only if the tangential component of its position vector field is a concurrent vector field. Moreover, rectifying submanifolds with arbitrary codimension are completely determined.

\section{Preliminaries}

Let $x: M \rightarrow \mathbb{E}^{m}$ be an isometric immersion of a Riemannian manifold $M$ into the Euclidean $m$-space $\mathbb{E}^{m}$. For each point $p \in M$, we denote by $T_{p} M$ and $T_{p}^{\perp} M$ the tangent and the normal spaces at $p$.

There is a natural orthogonal decomposition:

$$
T_{p} \mathbb{E}^{m}=T_{p} M \oplus T_{p}^{\perp} M .
$$

Denote by $\nabla$ and $\tilde{\nabla}$ the Levi-Civita connections of $M$ and $\mathbb{E}^{m}$, respectively. The formulas of Gauss and Weingarten are given respectively by (cf. [2, 9])

$$
\begin{aligned}
& \tilde{\nabla}_{X} Y=\nabla_{X} Y+h(X, Y), \\
& \tilde{\nabla}_{X} \xi=-A_{\xi} X+D_{X} \xi
\end{aligned}
$$

for vector fields $X, Y$ tangent to $M$ and $\xi$ normal to $M$, where $h$ is the second fundamental form, $D$ the normal connection, and $A$ the shape operator of $M$.

For a given point $p \in M$, the first normal space, of $M$ in $\mathbb{E}^{m}$, denoted by $\operatorname{Im} h_{p}$, is the subspace defined by

$$
\operatorname{Im} h_{p}=\operatorname{Span}\left\{h(X, Y): X, Y \in T_{p} M\right\} .
$$

For each normal vector $\xi$ at $p$, the shape operator $A_{\xi}$ is a self-adjoint endomorphism of $T_{p} M$. The second fundamental form $h$ and the shape operator $A$ are related by

$$
\left\langle A_{\xi} X, Y\right\rangle=\langle h(X, Y), \xi\rangle,
$$

where $\langle$,$\rangle is the inner product on M$ as well as on the ambient Euclidean space.

The equation of Gauss of $M$ in $\mathbb{E}^{m}$ is given by

$$
R(X, Y ; Z, W)=\langle\sigma(X, W), \sigma(Y, Z)\rangle-\langle\sigma(X, Z), \sigma(Y, W)\rangle
$$

for $X, Y, Z, W$ tangent to $M$, where $R$ denotes the curvature tensors of $M$.

The covariant derivative $\bar{\nabla} h$ of $h$ with respect to the connection on $T M \oplus T^{\perp} M$ is defined by

$$
\left(\bar{\nabla}_{X} h\right)(Y, Z)=D_{X}(h(Y, Z))-h\left(\nabla_{X} Y, Z\right)-h\left(Y, \nabla_{X} Z\right) .
$$

The equation of Codazzi is

$$
\left(\bar{\nabla}_{X} h\right)(Y, Z)=\left(\bar{\nabla}_{Y} h\right)(X, Z) .
$$

It follows from the definition of a rectifying curve $x: I \rightarrow \mathbb{E}^{3}$ that the position vector field $\mathrm{x}$ of $x$ satisfies

$$
\mathbf{x}(s)=\lambda(s) \mathbf{t}(s)+\mu(s) \mathbf{b}(s)
$$

for some functions $\lambda$ and $\mu$.

For a curve $x: I \rightarrow \mathbb{E}^{3}$ with $\kappa\left(s_{0}\right) \neq 0$ at $s_{0} \in I$, the first normal space at $s_{0}$ is the line spanned by the principal normal vector $\mathbf{n}\left(s_{0}\right)$. Hence, the rectifying plane at $s_{0}$ is nothing but the plane orthogonal to the first normal space at $s_{0}$. Therefore, for a submanifold $M$ of $\mathbb{E}^{m}$ and a point $p \in M$, we call the subspace of $T_{p} \mathbb{E}^{m}$, orthogonal complement to the first normal space $\operatorname{Im} \sigma_{p}$, the rectifying space of $M$ at $p$.

Definition 2.1. A submanifold $M$ of a Euclidean $m$-space $\mathbb{E}^{m}$ is called a rectifying submanifold if the position vector field $\mathrm{x}$ of $M$ always lies in its rectifying space. In other words, $M$ is called a rectifying submanifold if and only if

$$
\left\langle\mathbf{x}(p), \operatorname{Im} h_{p}\right\rangle=0
$$

holds at every $p \in M$. 
Definition 2.2. A non-trivial vector field $Z$ on a Riemannian manifold $M$ is called a concurrent vector field if it satisfies

$$
\nabla_{X} Z=X
$$

for any vector $X$ tangent to $M$, where $\nabla$ is the Levi-Civita connection of $M$.

\section{Lemmas}

By a cone in $\mathbb{E}^{m}$ with vertex at the origin we mean a ruled submanifold generated by a family of lines passing through the origin. A submanifold of $\mathbb{E}^{m}$ is called a conic submanifold with vertex at the origin if it is an open portion of a cone with vertex at the origin.

There exists a natural orthogonal decomposition of the position vector field $\mathrm{x}$ at each point for a Euclidean submanifold $M$; namely,

$$
\mathbf{x}=\mathbf{x}^{T}+\mathbf{x}^{N}
$$

where $\mathrm{x}^{T}$ and $\mathrm{x}^{N}$ denote the tangential and normal components of $\mathrm{x}$, respectively. Let $\left|\mathbf{x}^{T}\right|$ and $\left|\mathbf{x}^{N}\right|$ be the length of $\mathbf{x}^{T}$ and $\mathbf{x}^{N}$, respectively.

Lemma 3.1. Let $x: M \rightarrow \mathbb{E}^{m}$ be an isometric immersion of a Riemannian n-manifold into the Euclidean $m$-space $\mathbb{E}^{m}$. Then $\mathbf{x}=\mathbf{x}^{T}$ holds identically if and only if $M$ is a conic submanifold with the vertex at the origin.

Proof. Let $x: M \rightarrow \mathbb{E}^{m}$ be an isometric immersion of a Riemannian $n$-manifold into the Euclidean $m$-space $\mathbb{E}^{m}$. If $\mathbf{x}=\mathbf{x}^{T}$ holds identically, then $e_{1}=\mathbf{x} /|\mathbf{x}|$ is a unit vector field tangent to $M$.

Put $\mathbf{x}=\rho e_{1}$. Since $\tilde{\nabla}_{e_{1}} e_{1}$ is perpendicular to $e_{1}$, we find from

$$
\tilde{\nabla}_{e_{1}} \mathbf{x}=e_{1}, \quad \tilde{\nabla}_{e_{1}} \mathbf{x}=\left(e_{1} \rho\right) e_{1}+\rho \tilde{\nabla}_{e_{1}} e_{1},
$$

that $\tilde{\nabla}_{e_{1}} e_{1}=0$. Therefore, the integral curves of $e_{1}$ are some open portions of generating lines in $\mathbb{E}^{m}$. Moreover, because $\mathbf{x}=\mathbf{x}^{T}$, the generating lines given by the integral curves of $e_{1}$ pass through the origin. Consequently, $M$ is a conic submanifold with the vertex at the origin.

The converse is clear.

Lemma 3.2. Let $x: M \rightarrow \mathbb{E}^{m}$ be an isometric immersion of a Riemannian n-manifold into the Euclidean $m$-space $\mathbb{E}^{m}$. Then $\mathbf{x}=\mathbf{x}^{N}$ holds identically if and only if $M$ lies in a hypersphere centered at the origin.

Proof. Let $x: M \rightarrow \mathbb{E}^{m}$ be an isometric immersion of a Riemannian $n$-manifold into the Euclidean $m$-space $\mathbb{E}^{m}$. If $\mathbf{x}=\mathbf{x}^{N}$ holds identically, then we get

$$
Z\langle\mathbf{x}, \mathbf{x}\rangle=2\left\langle\tilde{\nabla}_{Z} \mathbf{x}, \mathbf{x}\right\rangle=2\left\langle Z, \mathbf{x}^{N}\right\rangle=0
$$

for any $Z \in T M$. Thus $M$ lies in a hypersphere centered at the origin.

The converse is obvious.

In views of Lemma 3.1 and Lemma 3.2 we make the following.

Definition 3.1. A rectifying submanifold $M$ of $\mathbb{E}^{m}$ is called proper if its position vector field $\mathbf{x}$ satisfies $\mathbf{x} \neq \mathbf{x}^{T}$ and $\mathbf{x} \neq \mathbf{x}^{N}$ at every point on $M$.

Lemma 3.3. Let $M$ be a proper rectifying submanifold of $\mathbb{E}^{m}$ with $\operatorname{dim} M=n$. Then we have

$$
m>n+\operatorname{dim}\left(\operatorname{Im} h_{p}\right)
$$

for each $p \in M$.

Proof. Let $M$ be a proper rectifying submanifold of $\mathbb{E}^{m}$. If $m=n+\operatorname{dim}\left(\operatorname{Im} h_{p}\right)$, then we get $\mathbf{x}=\mathbf{x}^{T}$ which is a contradiction.

Remark 3.1. In views of Lemma 3.1 and Lemma 3.2, we are only interested on proper rectifying submanifolds. 


\section{Characterization and classification of rectifying submanifolds}

First, we give the following simple characterization of rectifying submanifolds.

Theorem 4.1. If the position vector field $\mathbf{x}$ of a submanifold $M$ in $\mathbb{E}^{m}$ satisfies $\mathbf{x}^{N} \neq 0$, then $M$ is a proper rectifying submanifold if and only if $\mathbf{x}^{T}$ is concurrent vector field on $M$.

Proof. Let $x: M \rightarrow \mathbb{E}^{m}$ be an isometric immersion of a Riemannian $n$-manifold into the Euclidean $m$-space $\mathbb{E}^{m}$. Consider the orthogonal decomposition

$$
\mathbf{x}=\mathbf{x}^{T}+\mathbf{x}^{N}
$$

of the position vector field $\mathrm{x}$ of $M$ in $\mathbb{E}^{m}$.

From (4.1) and formulas of Gauss and Weingarten, we find

$$
Z=\tilde{\nabla}_{Z} \mathbf{x}=\nabla_{Z} \mathbf{x}^{T}+h\left(Z, \mathbf{x}^{T}\right)-A_{\mathbf{x}^{N}} Z+D_{Z} \mathbf{x}^{N}
$$

for any $Z \in T M$. After comparing the tangential components in (4.2), we obtain

$$
A_{\mathbf{x}^{N}} Z=\nabla_{Z} \mathbf{x}^{T}-Z \text {. }
$$

Assume that $M$ is a proper rectifying submanifold. Then we have $\mathbf{x}^{T} \neq 0$ and $\mathbf{x}^{N} \neq 0$. Moreover, it follows from the Definition 2.1 that

$$
\langle\mathbf{x}, h(X, Y)\rangle=0
$$

for $X, Y \in T M$. So we get $A_{\mathbf{x}^{N}}=0$. Hence, we obtain from (4.3) that

$$
\nabla_{Z} \mathbf{x}^{T}=Z,
$$

which shows that $\mathbf{x}^{T}$ is a concurrent vector field on $M$.

Conversely, if $\mathbf{x}^{T}$ is a concurrent vector field on $M$, then we find from (2.11) and (4.3) that $A_{\mathbf{x}^{N}}=0$. Therefore we obtain (4.4). Consequently, $M$ is a proper rectifying submanifold due to $\mathrm{x}^{N} \neq 0$ by assumption.

Next, we give the following classification of rectifying submanifolds.

Theorem 4.2. If $M$ is a proper rectifying submanifold of $\mathbb{E}^{m}$, then with respect to some suitable local coordinate systems $\left\{s, u_{2}, \ldots, u_{n}\right\}$ on $M$ the immersion $x$ of $M$ in $\mathbb{E}^{m}$ is of the form

$$
x\left(s, u_{2}, \ldots, u_{n}\right)=\sqrt{s^{2}+c^{2}} Y\left(s, u_{2}, \ldots, u_{n}\right), \quad\langle Y, Y\rangle=1, c>0,
$$

such that the metric tensor $g_{Y}$ of the spherical submanifold defined by $Y$ satisfies

$$
g_{Y}=\frac{c^{2}}{\left(s^{2}+c^{2}\right)^{2}} d s^{2}+\frac{s^{2}}{s^{2}+c^{2}} \sum_{i, j=2}^{n} g_{i j}\left(u_{2}, \ldots, u_{n}\right) d u_{i} d u_{j} .
$$

Conversely, the immersion given by (4.6)-(4.7) defines a proper rectifying submanifold.

Proof. Let $x: M \rightarrow \mathbb{E}^{m}$ be an isometric immersion of a Riemannian $n$-manifold $M$ into the Euclidean $m$-space $\mathbb{E}^{m}$. Assume that $M$ is a proper rectifying submanifold. Then (4.2) holds.

After comparing the normal components of (4.2), we obtain

$$
D_{Z} \mathbf{x}^{N}=-h\left(Z, \mathbf{x}^{T}\right)
$$

for $Z \in T M$.

It follows from (4.4) and (4.8) that $\left\langle\mathbf{x}, D_{Z} \mathbf{x}^{N}\right\rangle=0$. Hence we get

$$
Z\left\langle\mathbf{x}^{N}, \mathbf{x}^{N}\right\rangle=0,
$$

which implies that $\mathbf{x}^{N}$ is of positive constant length, say $c$. From (4.4) we obtain

$$
\left\langle A_{\mathbf{x}^{N}} X, Y\right\rangle=\left\langle\mathbf{x}^{N}, h(X, Y)\right\rangle=\langle\mathbf{x}, h(X, Y)\rangle=0 .
$$

www.iejgeo.com 
Hence we have $A_{\mathbf{x}^{N}}=0$. Let us put $\rho=\left|\mathbf{x}^{T}\right|$ and $e_{1}=\mathbf{x}^{T} / \rho$. We may extend $e_{1}$ to a local orthonormal frame $e_{1}, \ldots, e_{n}$.

We put

$$
\nabla_{X} e_{i}=\sum_{j=1}^{n} \omega_{i}^{j}(X) e_{j}, \quad i=1, \ldots, n
$$

For $j, k=2, \ldots, n$, we find

$$
0=e_{k}\left\langle\mathbf{x}, e_{j}\right\rangle=\delta_{j k}+\left\langle\mathbf{x}, \nabla_{e_{k}} e_{j}\right\rangle+\left\langle\mathbf{x}, h\left(e_{j}, e_{k}\right)\right\rangle
$$

Since $h\left(e_{j}, e_{k}\right)=h\left(e_{k}, e_{j}\right)$, equation (4.11) gives

$$
\omega_{j}^{1}\left(e_{k}\right)=\omega_{k}^{1}\left(e_{j}\right), \quad j, k=2, \ldots, n .
$$

Hence, it follows from the Frobenius theorem that the distribution $\mathcal{D}$ spanned by $e_{2}, \ldots, e_{n}$ is an integrable distribution.

On the other hand, the distribution $\mathcal{D}^{\perp}=\operatorname{Span}\left\{e_{1}\right\}$ is also integrable since it is of rank one. Therefore, there exist local coordinate systems $\left\{s, u_{2}, \ldots, u_{n}\right\}$ on $M$ such that $e_{1}=\partial / \partial s$ and $\partial / \partial u_{2}, \ldots, \partial / \partial u_{n}$ span the distribution $\mathcal{D}$.

Let us put

$$
\mathbf{x}^{T}=\varphi e_{1}
$$

with $\varphi=\left|\mathbf{x}^{T}\right|$. By taking the derivative of $\varphi=\left\langle\mathbf{x}, e_{1}\right\rangle$ with respect to $e_{j}$ for $j=1, \ldots, n$, we also have

$$
e_{j} \varphi=\delta_{1 j}+\left\langle\mathbf{x}, h\left(e_{1}, e_{j}\right)\right\rangle .
$$

Combining (4.4) and (4.13) gives

$$
e_{j} \varphi=\delta_{1 j}, \quad j=1, \ldots, n .
$$

Therefore, we obtain $\varphi=\varphi(s)$ and $\varphi^{\prime}(s)=1$ which imply $\varphi(s)=s+b$ for some constant $b$. Thus, after applying a suitable translation on $s$ if necessary, we have $\varphi=s$. Consequently, the position vector field satisfies

$$
\mathbf{x}=s e_{1}+\mathbf{x}^{N} .
$$

By combining (4.15) and $\left|\mathbf{x}^{N}\right|=c$, we find

$$
\langle\mathbf{x}, \mathbf{x}\rangle=s^{2}+c^{2},
$$

where $c$ is a positive number. Hence we may put

$$
x\left(s, u_{2}, \ldots, u_{n}\right)=\sqrt{s^{2}+c^{2}} Y\left(s, u_{2}, \ldots, u_{n}\right),
$$

for some $\mathbb{E}^{m}$-valued function $Y=Y\left(s, u_{2}, \ldots, u_{n}\right)$ satisfying $\langle Y, Y\rangle=1$.

Using (4.17) and the fact that $e_{1}=\partial / \partial s$ is orthogonal to the distribution $\mathcal{D}$, we obtain that

$$
\left\langle Y_{s}, Y_{s}\right\rangle=\frac{c^{2}}{\left(s^{2}+c^{2}\right)^{2}},\left\langle Y_{s}, Y_{u_{j}}\right\rangle=0, \quad j=2, \ldots, n
$$

Therefore, the metric tensor $g_{Y}$ of the spherical submanifold defined by $Y$ takes the following form:

$$
g_{Y}=\frac{c^{2}}{\left(s^{2}+c^{2}\right)^{2}} d s^{2}+\sum_{i, j=2}^{n} g_{i j}\left(s, u_{2}, \ldots, u_{n}\right) d u_{i} d u_{j} .
$$

On the other hand, it follows from Theorem 4.1 that $\mathrm{x}^{T}=s e_{1}$ is a concurrent vector field. Thus, we find from (4.5) that

$$
e_{1}=\nabla_{e_{1}} \mathbf{x}^{T}=\nabla_{e_{1}} s e_{1}=e_{1}+s \nabla_{e_{1}} e_{1} .
$$

Hence we get $\nabla_{e_{1}} e_{1}=0$, which implies that the integral curves of $e_{1}$ are geodesic in $M$. Therefore, the distribution $\mathcal{D}^{\perp}$ spanned by $e_{1}$ is a totally geodesic foliation.

From (4.5) we have

which implies that

$$
e_{i}=\nabla_{e_{i}} \mathbf{x}^{T}=s \nabla_{e_{i}} e_{1}, \quad i=2, \ldots, n,
$$

$$
\omega_{1}^{j}\left(e_{i}\right)=\frac{\delta_{i j}}{s}, i, j=2, \ldots, n
$$


where $\delta_{i j}=1$ or 0 depending on $i=j$ or $i \neq j$.

From (4.22) we conclude that $\mathcal{D}$ is an integrable distribution whose leaves are totally umbilical in $M$. Moreover, the mean curvature of leaves of $\mathcal{D}$ are given by $s^{-1}$. Since the leaves of $\mathcal{D}$ are hypersurfaces in $M$, it follows that the mean curvature vector fields of leaves of $\mathcal{D}_{2}$ are parallel in the normal bundle of $M$ in $\mathbb{E}^{m}$. Therefore, $\mathcal{D}$ is a spherical foliation. Consequently, by a result of [13] (or Theorem 4.4 of [9, page 90]) we conclude that $M$ is locally a warped product $I \times{ }_{s} F$, where $F$ is a Riemannian $(n-1)$-manifold. Thus, the metric tensor $g$ of $M$ takes the form

$$
g=d s^{2}+s^{2} g_{F},
$$

where $g_{F}$ is the metric tensor of $F$. Now, by applying (4.6), (4.19) and (4.23), we may conclude that the metric tensor $g_{Y}$ can be expressed as (4.7).

Conversely, let us consider a submanifold $M$ of $\mathbb{E}^{m}$ defined by

$$
x\left(s, u_{2}, \ldots, u_{n}\right)=\sqrt{s^{2}+c^{2}} Y\left(s, u_{2}, \ldots, u_{n}\right), \quad\langle Y, Y\rangle=1, c>0,
$$

such that the metric tensor $g_{Y}$ satisfies

$$
g_{Y}=\frac{c^{2}}{\left(s^{2}+c^{2}\right)^{2}} d s^{2}+\frac{s^{2}}{s^{2}+c^{2}} \sum_{i, j=2}^{n} g_{i j}\left(u_{2}, \ldots, u_{n}\right) d u_{i} d u_{j} .
$$

Then it follows from (4.24) that

$$
\begin{aligned}
& \frac{\partial \mathbf{x}}{\partial s}=\frac{s Y}{\sqrt{s^{2}+c^{2}}}+\sqrt{s^{2}+c^{2}} Y_{s}, \\
& \frac{\partial \mathbf{x}}{\partial u_{j}}=\sqrt{s^{2}+c^{2}} Y_{u_{j}}, \quad j=2, \ldots, n,
\end{aligned}
$$

where $Y_{s}=\partial Y / \partial s$ and $Y_{u_{j}}=\partial Y / \partial u_{j}$. It follows from (4.24), (4.25) and (4.26) that the metric tensor $g_{M}$ of $M$ is given by

$$
g_{M}=d s^{2}+s^{2} \sum_{i, j=2}^{n} g_{i j}\left(u_{2}, \ldots, u_{n}\right) d u_{i} d u_{j} .
$$

Now, by an easy computation, we find from (4.27) that

$$
\nabla_{\frac{\partial}{\partial s}} \frac{\partial}{\partial s}=0, \quad \nabla_{\frac{\partial}{\partial u_{j}}} \frac{\partial}{\partial s}=\frac{1}{s} \frac{\partial}{\partial u_{j}}, j=2, \ldots, n .
$$

Since $\langle Y, Y\rangle=1,(4.24)$ and (4.26) imply that

$$
\left\langle\mathbf{x}, \mathbf{x}_{u_{j}}\right\rangle=0, \quad j=2, \ldots, n .
$$

Therefore, we obtain $\mathbf{x}^{T}=s \frac{\partial}{\partial s}$. Now, it is easy to verify that $\mathbf{x}^{T}$ is a concurrent vector field on $M$. Moreover, it is direct to show that the normal component of $\mathrm{x}$ is given by

$$
\mathbf{x}^{N}=\frac{c^{2}}{\sqrt{s^{2}+c^{2}}} Y-s \sqrt{s^{2}+c^{2}} Y_{s},
$$

which is alway non-zero everywhere on $M$. Consequently, $M$ is a proper rectifying submanifold, according to Theorem 4.1.

Remark 4.1. Theorem 4.2 extend Theorem 3 of [6].

Remark 4.2. If we put $s=\tan ^{-1}\left(\frac{t}{c}\right)$, then (4.7) becomes

$$
g_{Y}=d t^{2}+\sin ^{2} t \sum_{j, k=2}^{n} g_{j k}\left(u_{2}, \ldots, u_{n}\right) d u_{j} d u_{k} .
$$

For $n=2$, we get $g_{Y}=d t^{2}+\left(\sin ^{2} t\right) d u^{2}$ from (4.30), which is the metric tensor of a spherical coordinate system $(t, u)$ on $S^{2}(1)$. Hence, for $n=2, Y=Y(t, u)$ is nothing but an isometric immersion from an open portion of $S^{1}(1)$ into $S^{m-1}(1) \subset \mathbb{E}^{m}$. Therefore, there exist many spherical submanifolds in $\mathbb{E}^{m}$ whose metric tensor is given by (4.7). Consequently, there exist many rectifying submanifolds in $\mathbb{E}^{m}$ according to Theorem 4.2. 


\section{Some properties of rectifying submanifolds}

Finally, we provide some basic properties of proper rectifying submanifolds.

Theorem 5.1. Let $M$ be a proper rectifying submanifold of $\mathbb{E}^{m}$. Then

(a) $\left|\mathbf{x}^{T}\right|=s+b$ for some constant $b$.

(b) $|\mathbf{x}|^{2}=s^{2}+c_{1} s+c_{2}$ for some constants $c_{1}$ and $c_{2}$.

(c) $\mathbf{x}^{N}$ is of constant length.

(d) $A_{\mathbf{x}^{N}}=0$.

(e) The curvature tensor $R$ satisfies $R\left(\mathbf{x}^{T}, Y\right)=0$ for any $Y \in T M$.

(f) The sectional curvature $K$ of $M$ satisfies $K\left(\mathbf{x}^{T}, Z\right)=0$ for any unit vector $Z$ perpendicular to $\mathbf{x}^{T}$.

Proof. Statements (a), (b), (c) and (d) are already done in the proof of Theorem 4.2. Clearly, statement (f) follows immediately from statement (e).

Now, we prove statement (e). This can be done as follows. By applying (2.6) and (4.8) we have

$$
\begin{aligned}
R\left(\mathbf{x}^{T}, Y, Z ; W\right) & =\left\langle h\left(\mathbf{x}^{T}, W\right), h(Y, Z)\right\rangle-\left\langle h\left(\mathbf{x}^{T}, Z\right), h(Y, W)\right\rangle \\
& =\left\langle D_{Z} \mathbf{x}^{N}, h(Y, W)\right\rangle-\left\langle D_{W} \mathbf{x}^{N}, h(Y, Z)\right\rangle \\
& =-\left\langle\mathbf{x}^{N}, D_{Z} h(Y, W)\right\rangle+\left\langle\mathbf{x}^{N}, D_{W} h(Y, Z)\right\rangle .
\end{aligned}
$$

Therefore, after applying (4.4) and equation (2.8) of Codazzi, we derive from (5.1) that

$$
R\left(\mathbf{x}^{T}, Y, Z ; W\right)=\left\langle\mathbf{x}^{N},\left(\bar{\nabla}_{W} h\right)(Y, Z)\right\rangle-\left\langle\mathbf{x}^{N},\left(\bar{\nabla}_{Z} h\right)(Y, W)\right\rangle=0
$$

which gives statement (e).

Remark 5.1. Statement (a), (b) and (c) of Theorem 5.1 extend the corresponding results obtained in Theorem 1 of [6].

Remark 5.2. One may define rectifying submanifolds in a pseudo-Euclidean space in the same as Definition 2.1. We will treat rectifying submanifolds in pseudo-Euclidean spaces in a separate article.

\section{References}

[1] Cambie, S., Goemans, W. and Van den Bussche, I., Rectifying curves in the $n$-dimensional Euclidean space. Turkish J. Math. 40 (2016), no. $1,210-223$

[2] Chen, B.-Y., Geometry of Submanifolds. Marcel Dekker, New York, 1973.

[3] Chen, B.-Y., Constant-ratio hypersurfaces. Soochow J. Math. 21 (2001), 353-361.

[4] Chen, B.-Y., Geometry of position functions of Riemannian submanifolds in pseudo-Euclidean space. J. Geom. 74 (2002), 61-77.

[5] Chen, B.-Y., Convolution of Riemannian manifolds and its applications. Bull. Austral. Math. Soc. 66 (2002), no. 2, 177-191.

[6] Chen, B.-Y., When does the position vector of a space curve always lie in its rectifying plane?. Amer. Math. Monthly 110 (2003), no. 2, $147-152$.

[7] Chen, B.-Y., More on convolution of Riemannian manifolds. Beiträge Algebra Geom. 44 (2003), 9-24.

[8] Chen, B.-Y., Constant-ratio space-like submanifolds in pseudo-Euclidean space. Houston J. Math. 29 (2003), no. 2, 281-294

[9] Chen, B.-Y., Pseudo-Riemannian geometry, $\delta$-invariants and applications. World Scientific, 2011.

[10] Chen, B.-Y., Topics in differential geometry associated with position vector fields on Euclidean submanifolds. Arab J. Math. Sci. 23 (2017), no. 1 (Special Issue on Geometry and Global Analysis), doi:10.1016/j.ajmsc.2016.08.001.

[11] Chen, B.-Y. and Dillen, F., Rectifying curves as centrodes and extremal curves. Bull. Inst. Math. Acad. Sinica 33 (2005), no. 2, 77-90.

[12] Gungor, M. A. and Tosun, M., Some characterizations of quaternionic rectifying curves. Differ. Geom. Dyn. Syst. 13 (2011), 89-100.

[13] S. Hiepko, Eine innere Kennzeichnung der verzerrten Produkte, Math. Ann. 241 (1979), no. 3, 209-215.

[14] Ilarslan, K., Nesovic, E. and Petrovic-Torgasev, M., Some characterizations of rectifying curves in the Minkowski 3-space. Novi Sad J. Math. 33 (2003), no. 2, 23-32.

[15] Ilarslan, K. and Nesovic, E., On rectifying curves as centrodes and extremal curves in the Minkowski 3-space. Novi Sad J. Math. 37 (2007), no. 1, 53-64.

[16] Ilarslan, K. and Nesovic, E., Some characterizations of rectifying curves in the Euclidean space $\mathbb{E}^{4}$. Turkish J. Math. 32 (2008), no. 1, 21-30.

[17] Ilarslan, K. and Nesovic, E., Some relations between normal and rectifying curves in Minkowski space-time. Int. Electron. J. Geom. 7 (2014), no. 1, 26-35.

[18] Lucas, P. and Ortega-Yagues, J. A., Rectifying curves in the three-dimensional sphere. J. Math. Anal. Appl. 421 (2015), no. 2, $1855-1868$.

[19] Ozbey, and Oral, M., A study on rectifying curves in the dual Lorentzian space. Bull. Korean Math. Soc. 46 (2009), no. 5, 967-978.

[20] Yilmaz, B., Gok, I. and Yayli, Y., Extended rectifying curves in Minkowski 3-space. Adv. Appl. Clifford Algebr. 26 (2016), no. 2, 861-872.

[21] Yücesan, A., Ayyildiz, N. and Coken, A. C., On rectifying dual space curves. Rev. Mat. Complut. 20 (2007), no. 2, 497-506. 


\section{Affiliations}

BANG-YEN CHEN

AdDREss: Michigan State University, Department of Mathematics, 619 Red Cedar Road, East Lansing, Michigan 48824-1027, U.S.A.

E-MAIL: bychen@math.msu.edu 